\title{
CONTRIBUTIONS TO A CONJECTURE OF MUELLER AND SCHMIDT ON THUE INEQUALITIES
}

\author{
N. SARADHA AND DIVYUM SHARMA
}

Abstract. Let $F(X, Y)=\sum_{i=0}^{s} a_{i} X^{r_{i}} Y^{r-r_{i}} \in \mathbb{Z}[X, Y]$ be a form of degree $r=r_{s} \geq 3$, irreducible over $\mathbb{Q}$ and having at most $s+1$ nonzero coefficients. Mueller and Schmidt showed that the number of solutions of the Thue inequality

$$
|F(X, Y)| \leq h
$$

is $\ll s^{2} h^{2 / r}\left(1+\log h^{1 / r}\right)$. They conjectured that $s^{2}$ may be replaced by $s$. Let

$$
\Psi=\max _{0 \leq i \leq s} \max \left(\sum_{w=0}^{i-1} \frac{1}{r_{i}-r_{w}}, \sum_{w=i+1}^{s} \frac{1}{r_{w}-r_{i}}\right) .
$$

Then we show that $s^{2}$ may be replaced by $\max \left(s \log ^{3} s, s e^{\Psi}\right)$. We also show that if $\left|a_{0}\right|=\left|a_{s}\right|$ and $\left|a_{i}\right| \leq\left|a_{0}\right|$ for $1 \leq i \leq s-1$, then $s^{2}$ may be replaced by $s \log ^{3 / 2} s$. In particular, this is true if $a_{i} \in\{-1,1\}$.

\section{INTRODUCTION}

1.1. Thue inequalities for forms with few coefficients. Let $F(X, Y)$ be a form of degree $r \geq 3$ with integer coefficients, irreducible over $\mathbb{Q}$ and having at most $s+1$ non-zero coefficients. Write

$$
F(X, Y)=\sum_{i=0}^{s} a_{i} X^{r_{i}} Y^{r-r_{i}}
$$

with $0=r_{0}<r_{1}<\ldots<r_{s}=r$. Let $D, H$ and $M$ denote the discriminant, height and Mahler height of $F(X, 1)$ respectively. For $h \geq 1$, consider the Thue inequality

$$
|F(X, Y)| \leq h
$$

2010 Mathematics Subject Classification. Primary 11J25, 11D59 ; Secondary $11 \mathrm{D} 45$.

Key words and phrases. Thue equations, Thue inequalities, Archimedean Newton polygon. 
Let $N_{F}(h)$ denote the number of integer solutions $(x, y)$ of (2). Schmidt [19] proved that

$$
N_{F}(h) \ll \sqrt{r s} h^{2 / r}\left(1+\log h^{1 / r}\right) .
$$

Prior to this result, Bombieri and Schmidt [3] considered the equation

$$
|F(X, Y)|=h .
$$

They showed that

$$
N_{F}^{(1)}(h) \ll r^{1+\omega(h)}
$$

where $N_{F}^{(1)}(h)$ denotes the number of solutions $(x, y)$ of (4i) with $\operatorname{gcd}(x, y)$ $=1$ and $\omega(h)$ denotes the number of distinct prime divisors of $h$. Since $s \leq r$, it can be easily seen that (3) is better than (15) if $h$ is given and $r$ is large while (5) is better than (3) if $r$ is given and $h$ is large. The proof of inequality (15) is based on the Thue-Siegel principle and $p$-adic arguments. Schmidt's result depended also on the analysis of location of roots of $F(X, 1)$. Bombieri modified a conjecture of Siegel on the inequality (2) as

$$
N_{F}(h) \leq C(s, h)
$$

where $C(s, h)$ depends only on $s$ and $h$. (See Mueller and Schmidt [18, p. 208]). This was shown to be true in the case $s=1$ by Hyyrö [10], Evertse [6] and Mueller [16]. The case $s \geq 2$ was considered by Mueller and Schmidt in [17] and [18. They proved that

$$
N_{F}(h) \ll s^{2} C_{1}(r, h)
$$

where $C_{1}(r, h)=h^{2 / r}\left(1+\log h^{1 / r}\right)$. In all the estimates for $N_{F}(h)$, the factor $h^{2 / r}$ in $C_{1}(r, h)$ is unavoidable. The logarithmic factor in $C_{1}(r, h)$ was improved by Thunder when $h$ is large, see [20] and [21]. In fact, in [22], Thunder proved a general result on the number of solutions of decomposable form inequalities (higher dimensional Thue inequalities), which implies that for Thue inequalities (2), we have

$$
N_{F}(h) \leq C_{2}(r) h^{2 / r},
$$

where $C_{2}(r)$ is an effectively computable, but inexplicit number depending only on $r$. Further, it is expected that $N_{F}(h)$ is close to $A_{F} h^{2 / r}$, where $A_{F}$ is the area of the set of $(\xi, \eta) \in \mathbb{R}^{2}$ with $|F(\xi, \eta)| \leq 1$. In [14], Mahler showed the finiteness of $A_{F}$. Later, Mueller \& Schmidt [18] and Bean [2] gave explicit upper estimates for $A_{F}$. For instance, for any form $F$ with non-zero discriminant, Bean showed that

$$
A_{F} \leq 3 B\left(\frac{1}{3}, \frac{1}{3}\right)<16
$$


where $B(m, n)$ is the classical beta function. The bound $3 B\left(\frac{1}{3}, \frac{1}{3}\right)$ is attained for forms which are equivalent under $S L_{2}(\mathbb{R})$ to $X Y(X-Y)$. We refer to Evertse \& Györy [7] and Györy [9] for more results on Thue equations and Thue inequalities.

It was conjectured in [18 that it may be possible to replace the factor $s^{2}$ in (6) by $s$. In Theorem 1.1 below, we show that when the nonzero terms of $F$ are sufficiently far apart, then $s^{2}$ can be improved. In Theorem 1.5, we improve (6) for forms with restricted coefficients.

Theorem 1.1. Let $F(X, Y)$ be given by (1). Put

$$
\Psi=\max _{0 \leq i \leq s} \max \left(\sum_{w=0}^{i-1} \frac{1}{r_{i}-r_{w}}, \sum_{w=i+1}^{s} \frac{1}{r_{w}-r_{i}}\right)
$$

and

$$
\Phi=\max (\Psi, 3 \log \log s)
$$

Then we have

$$
N_{F}(h) \ll s e^{\Phi} C_{1}(r, h) .
$$

(In (7), an empty sum is taken to be equal to zero.)

Remark 1.2. Suppose $r \leq 4 s e^{2 \Phi}$. Then (3) implies (8). Thus, for proving Theorem 1.1, we may assume that

$$
r>4 s e^{2 \Phi}
$$

Further, we may take $s \gg 1$, as otherwise inequality (6) is sufficient.

Throughout the paper, $c_{1}, c_{2}, \ldots$ denote positive absolute constants. We shall illustrate Theorem 1.1] with some examples below.

Remark 1.3. Let $0 \leq i, w \leq s, i \neq w$. Since $\left|r_{i}-r_{w}\right| \geq|i-w|$, it follows that

$$
\Psi \leq \max _{0 \leq i \leq s} \max \left(\sum_{n=1}^{i} \frac{1}{n}, \sum_{n=1}^{s-i} \frac{1}{n}\right) \leq \log s+c_{1},
$$

(see [1, p. 55]). Thus we get (6) .

Remark 1.4. We give some instances when $s^{2}$ in (6) can be improved.

(i) Suppose $\left|r_{i}-r_{w}\right| \geq c_{2}|i-w|$ with $c_{2}>1$. Then $\Psi \leq \frac{1}{c_{2}} \log s+c_{3}$. Hence

$$
N_{F}(h) \ll s^{1+\frac{1}{c_{2}}} C_{1}(r, h) .
$$


(ii) Suppose $\left|r_{i}-r_{w}\right| \geq \frac{1}{3}|i-w| \log |i-w|$. Then

$$
\Psi \leq 1+\max _{0 \leq i \leq s} \max \left(\sum_{n=2}^{i} \frac{3}{n \log n}, \sum_{n=2}^{s-i} \frac{3}{n \log n}\right) \leq 3 \log \log s+c_{4},
$$

(see [1, p. 70]). Hence

$$
N_{F}(h) \ll s \log ^{3} s C_{1}(r, h) .
$$

In a different direction, we impose restrictions on the coefficients of $F$ and improve ([6) $)$.

Theorem 1.5. Suppose that the coefficients of $F(X, Y)$ satisfy

$$
\left|\frac{a_{0}}{a_{s}}\right|^{1 / r_{s}} \leq\left|\frac{a_{0}}{a_{i}}\right|^{1 / r_{i}} \text { for } i=1, \ldots, s-1 .
$$

If $r \geq s \log ^{3} s$, then

$$
N_{F}(h) \ll s(\log s) h^{2 / r} .
$$

Remark 1.6. If $r<s \log ^{3} s$, then by (3) we have

$$
N_{F}(h) \ll s \log ^{3 / 2} s C_{1}(r, h) .
$$

As an immediate consequence of Theorem 1.5 and Remark 1.6 we get

Corollary 1.7. Suppose that $\left|a_{0}\right|=\left|a_{s}\right|=H$, where $H$ is the height of F. Then

$$
N_{F}(h) \ll s \log ^{3 / 2} s C_{1}(r, h) .
$$

In particular, the above estimate is valid if the coefficients $a_{i}$ assume only the values \pm 1 .

Theorem 1.1 is a consequence of the following result.

Proposition 1.8. Let $F(X, Y)$ be given by (1). Then

$$
N_{F}(h) \ll s\left(\frac{\log s}{\Phi}+e^{\Phi+c_{5}\left(\log ^{3} s\right) e^{-\Phi}}\right) C_{1}(r, h) .
$$

Let $X_{1}$ and $X_{2}$ be positive numbers. Divide the solutions $(x, y)$ of (2) into three sets according as

$$
\begin{gathered}
\max (|x|,|y|)>X_{1} ; \max (|x|,|y|) \leq X_{1} \text { and } \min (|x|,|y|) \geq X_{2} \\
\min (|x|,|y|)<X_{2} .
\end{gathered}
$$

In [18], the solutions in these sets were called large, medium and small respectively. Denote the number of primitive solutions, i.e. solutions $(x, y)$ with $\operatorname{gcd}(x, y)=1$, in these sets by $P_{\ell a r}\left(X_{1}\right), P_{m e d}\left(X_{1}, X_{2}\right)$ and $P_{\text {sma }}\left(X_{2}\right)$ respectively. If $X_{2}>X_{1}$, put $P_{\text {med }}\left(X_{1}, X_{2}\right)=0$. We can 
bound $N_{F}(h)$ by finding estimates for these quantities (See Section 9). Mueller and Schmidt had shown that

$$
P_{\text {lar }}\left(Y_{W}\right) \ll s
$$

where $Y_{W}$ is as given in (12) below. In Theorem 1.9)(i), we have lowered the value of $Y_{W}$ and obtained the same conclusion as in (11). For medium solutions, we analyze precisely the large derivatives which play an important role in obtaining good rational approximations. (See Lemma 5.6). The small solutions are handled as in [18].

1.2. Large solutions. It was noted by Brindza, Pintér, van der Poorten \& Waldschmidt [5] that it seems likely that almost all the solutions of (4) are small, and around $h^{1 / r}$ provided that $h$ is large compared to $H$. In this section, we present some results supporting this observation. We use some notations from [3]. Choose numbers $a, b$ with $0<a<b<1$. Define

$$
\begin{gathered}
t=\sqrt{2 /\left(r+a^{2}\right)}, \lambda=2 /((1-b) t), \\
\delta=\frac{\left(r+b^{2}\right) t^{2}-2}{r-1}, A=\frac{1}{a^{2}}\left(\log M+\frac{r}{2}\right) .
\end{gathered}
$$

Further, we put

$$
\begin{gathered}
B=\frac{2^{r} r^{r / 2} M^{r} h}{\sqrt{|D|}}, \\
Y_{E}=(2 B \sqrt{|D|})^{1 /(r-\lambda)}\left(4 e^{A}\right)^{\lambda /(r-\lambda)} \\
Y_{G}=(2 B)^{\frac{1}{r-2}+\frac{1}{r^{2}}} \text { and } Y_{W}=R_{1}^{1 /(r-\lambda)} Y_{E}
\end{gathered}
$$

where

$$
R_{1}=e^{800 \log ^{3} r} .
$$

Since $|D| \leq r^{r} M^{2 r-2}([13$, Theorem 1]), we have $B>1$. We use this notation without any further mention in the rest of the paper. It was shown by Bombieri \& Schmidt [3] that $P_{\ell a r}\left(Y_{E}\right) \ll r$. Improving upon [5], Györy [8] showed that $P_{\ell a r}\left(Y_{G}\right) \leq 25 r$. (In fact, he proved that $P_{\ell a r}\left(Y_{G}\right) \leq 5 r$ if $r$ is sufficiently large.) Since $Y_{G}$ is much smaller than $Y_{E}$, the latter result is better than the former. Further, Schmidt [19] showed that

$$
P_{\ell a r}\left(Y_{E}\right) \ll \sqrt{r s}
$$

and as mentioned already in (11), Mueller \& Schmidt obtained that

$$
P_{\text {lar }}\left(R_{1}^{1 /(r-\lambda)} Y_{E}\right) \ll s .
$$

We shall improve the result of Györy mentioned above in the following theorem. Further, using a result of Mignotte [15] on the distribution of zeros of polynomials (see Lemma 3.2), we give different upper bounds for large primitive solutions. 
Theorem 1.9. Let $F(X, Y)$ be as in (1). Then

(i) $P_{\text {lar }}\left(Y_{G} R_{1}^{\frac{1}{r-2}+\frac{1}{r^{2}}}\right) \ll s$.

(ii) Put

Then

$$
\Delta=\frac{\sqrt{3|D|}}{2 r^{\frac{r+2}{2}} M^{r-1}} \text { and } R_{2}=1+\frac{M r}{2 \Delta} .
$$

$$
P_{\ell a r}\left(Y_{G} R_{2}^{\frac{1}{r-2}+\frac{1}{r^{2}}}\right) \ll \sqrt{r(\log r+\log M)} .
$$

Remark 1.10. Note that

$$
Y_{G} R_{1}^{\frac{1}{r-2}+\frac{1}{r^{2}}} \ll Y_{G}
$$

and

$$
Y_{G} R_{2}^{\frac{1}{r-2}+\frac{1}{r^{2}}} \ll Y_{G}^{2}
$$

When the coefficients $a_{i}$ of $F$ assume only the values \pm 1 , then by a result of Borwein and Erdelyi [4], there can be $\ll \sqrt{r}$ roots in the strip $|\operatorname{Im}(z)| \leq \frac{2}{\sqrt{r}}$. Using this fact in place of the result of Mignotte, it is possible to show that

$$
P_{\ell a r}\left(Y_{G} R_{3}^{\frac{1}{r-2}+\frac{1}{r^{2}}}\right) \ll \sqrt{r}
$$

where

Note also that

$$
R_{3}=1+M \sqrt{r}
$$

$$
Y_{G} R_{3}^{\frac{1}{r-2}+\frac{1}{r^{2}}} \ll Y_{G}^{2}
$$

\section{Counting elements of A Set}

On many occasions, we need to estimate the cardinality of a set whose elements satisfy certain properties which are termed as gap principle in the theory of Thue equations. In the following lemma, we give two instances in a formal setup.

Lemma 2.1. Let $n \geq 2$ and let $U=\left\{u_{1}, \ldots, u_{n}\right\}$ be a set together with a map $T: U \rightarrow \mathbb{R}^{*}$ such that

$$
A_{1} \leq T\left(u_{1}\right) \leq T\left(u_{2}\right) \leq \ldots \leq T\left(u_{n}\right)
$$

and

$$
T\left(u_{i}\right) \geq \beta T\left(u_{i-1}\right)^{\gamma} \text { for } 2 \leq i \leq n,
$$

where $\beta>0, \gamma \geq 2$. Let

$$
\kappa= \begin{cases}2 & \text { if } \beta>1 \\ 1 & \text { if } \beta \leq 1\end{cases}
$$


(i) Suppose that $T\left(u_{n}\right) \leq B_{1}$ and $A_{1} \beta^{1 /(\kappa(\gamma-1))}>1$. Then

$$
n \leq 1+\frac{1}{\log \gamma} \log \left(\frac{\log B_{1}}{\log A_{1}+(\log \beta) /(\kappa(\gamma-1))}\right) .
$$

(ii) Suppose that

$$
T\left(u_{n}\right) \leq\left(\eta_{1} T\left(u_{1}\right)\right)^{\eta_{2}}
$$

with $\eta_{1}>1,1<\eta_{2} \leq \gamma^{n-1}$. Let $\beta \leq 1$ and $A_{1} \geq\left(\frac{\eta_{1}^{\mu}}{\beta}\right)^{1 / \nu}$ with $1 \leq \mu<\nu<\gamma-1$. Then

$$
n \leq 1+\frac{1}{\log \gamma} \log \left(\eta_{2} \max \left(\frac{\mu+\nu}{\mu}, \frac{1}{1-\nu /(\gamma-1)}\right)\right) \text {. }
$$

Proof. From (14), by induction, we get

$$
\begin{aligned}
T\left(u_{n}\right) & \geq \beta^{1+\gamma+\ldots+\gamma^{n-2}} T\left(u_{1}\right)^{\gamma^{n-1}} \\
& \geq\left(\beta^{1 /(\kappa(\gamma-1))} T\left(u_{1}\right)\right)^{\gamma^{n-1}} .
\end{aligned}
$$

(i) Since $T\left(u_{n}\right) \leq B_{1}$, (18) implies that

$$
1<\left(\beta^{1 /(\kappa(\gamma-1))} T\left(u_{1}\right)\right)^{\gamma^{n-1}} \leq B_{1} .
$$

Taking logarithm and using $T\left(u_{1}\right) \geq A_{1}$, we find

$$
\gamma^{n-1} \leq \frac{\log B_{1}}{\log A_{1}+(\log \beta) /(\kappa(\gamma-1))}
$$

Since $\gamma \geq 2, n \geq 2$, the right hand side of the above inequality is $>1$. Taking logarithm once again, we get (15).

(ii) From (16) and (18), we get

$$
\left(\beta^{1 /(\gamma-1)} T\left(u_{1}\right)\right)^{\gamma^{n-1}} \leq\left(\eta_{1} T\left(u_{1}\right)\right)^{\eta_{2}}
$$

Taking logarithms and using $\eta_{2} \leq \gamma^{n-1}, T\left(u_{1}\right) \geq A_{1} \geq\left(\frac{\eta_{1}^{\mu}}{\beta}\right)^{1 / \nu}$, we get

$$
\begin{aligned}
\gamma^{n-1} & \leq \frac{\eta_{2}\left(\left(1+\frac{\mu}{\nu}\right) \log \eta_{1}+\frac{1}{\nu} \log \frac{1}{\beta}\right)}{\frac{\mu}{\nu} \log \eta_{1}+\left(\frac{1}{\nu}-\frac{1}{\gamma-1}\right) \log \frac{1}{\beta}} \\
& \leq \eta_{2} \max \left(\frac{\mu+\nu}{\mu}, \frac{1}{1-\nu /(\gamma-1)}\right)
\end{aligned}
$$

since $\frac{\rho_{1}+\rho_{2}}{\rho_{3}+\rho_{4}} \leq \max \left(\frac{\rho_{1}}{\rho_{3}}, \frac{\rho_{2}}{\rho_{4}}\right)$ for positive values of $\rho_{1}, \rho_{2}, \rho_{3}, \rho_{4}$. Again taking logarithms in the above inequality, we get (17). 


\section{A small Set of Roots Close to solutions}

Let $S$ be any finite set of complex numbers and let $\xi$ be a real number. Define the distance of $\xi$ from $S$, denoted by $d(S, \xi)$, as

$$
d(S, \xi)=\min _{\eta \in S}|\xi-\eta|
$$

Thus if $\xi \in S$, then $d(S, \xi)=0$. In the ensuing discussions, we specialize $S$ as the set of roots $\alpha_{1}, \ldots, \alpha_{r}$ of

$$
f(Z)=F(Z, 1)
$$

These discussions are valid if we replace $S$ by $S^{*}$, which is the set of roots $\beta_{1}, \ldots, \beta_{r}$ of $F(1, Z)$. Note that $\left\{\beta_{1}, \ldots, \beta_{r}\right\}=\left\{\alpha_{1}^{-1}, \ldots, \alpha_{r}^{-1}\right\}$ and $F(1, Z)$ has the same discriminant, height and Mahler height as $F(Z, 1)$. In [18], the following result was shown.

Lemma 3.1. [18, Lemma 7]

There is a set $S_{1} \subseteq S$ with $\left|S_{1}\right| \leq 6 s+4$ such that for any real $\xi$, we have

$$
d\left(S_{1}, \xi\right) \leq R_{1} d(S, \xi)
$$

where $R_{1}$ is given by (13).

We now state a lemma on the distribution of roots of a polynomial due to Mignotte [15].

Lemma 3.2. Let $P$ be an irreducible polynomial of degree $d$, with integer coefficients. Let $V$ be a sector (open or closed) in the complex plane, centred at the origin with central angle $2 \pi \theta$, where $0 \leq \theta \leq 1$. Then the number $N(V)$ of roots of $P$ in $V$ satisfies

$$
N(V) \leq 2 \theta d+c_{6} \sqrt{2 d(1.5 \log (2 d)+2 \log M(P))},
$$

where $M(P)$ is the Mahler height of $P$.

We apply Lemma 3.2 to get a subset of $S$ having a property similar to the set $S_{1}$ in Lemma 3.1 .

Lemma 3.3. There is a set $S_{2} \subseteq S$ with $\left|S_{2}\right| \ll \sqrt{r(\log r+\log M)}$ such that for any real $\xi$, we have

$$
d\left(S_{2}, \xi\right) \leq R_{2} d(S, \xi)
$$

where $R_{2}$ is as given in Theorem 1.9(ii).

Proof. Define

$$
\Delta(f)=\min _{1 \leq i<j \leq r}\left|\alpha_{i}-\alpha_{j}\right| .
$$

By [13, Theorem 2], we have

$$
\Delta(f)>2 \Delta
$$


where $\Delta$ is as in Theorem 1.9)(ii).

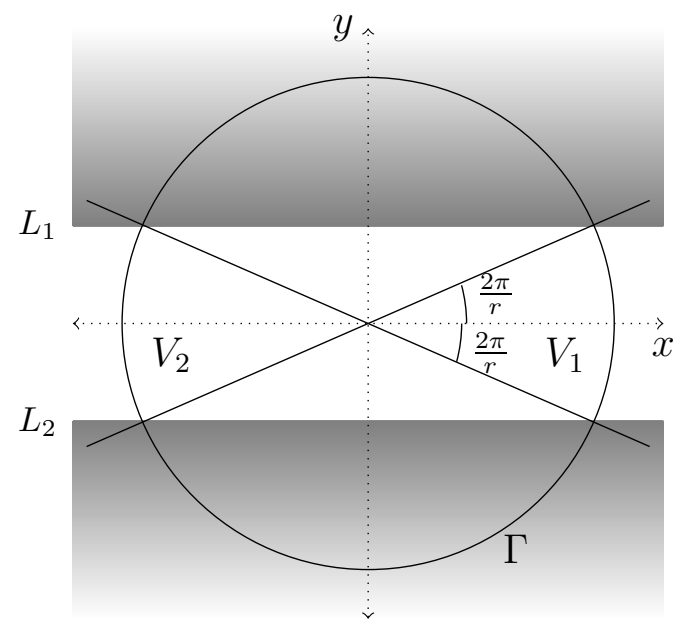

FiguRE 1.

Therefore there can be at most one root of $f$ inside the circle $\Gamma$ centred at the origin and having radius $\Delta$. Now consider the sector $V_{1}$ shown in Figure 1. It is centred at the origin and has central angle $4 \pi / r$. Let $N\left(V_{1}\right)$ denote the number of zeros of $f$ inside the sector $V_{1}$. By Lemma 3.2 with $M(P)=M(f)=M$, we have

$$
N\left(V_{1}\right) \leq 4+c_{6} \sqrt{2 r(1.5 \log (2 r)+2 \log M)} \ll \sqrt{r(\log r+\log M)} .
$$

We also have $N\left(V_{2}\right) \ll \sqrt{r(\log r+\log M)}$. If $m$ denotes the number of roots inside $V_{1} \cup V_{2} \cup \Gamma$, then $m \ll \sqrt{r(\log r+\log M)}$. The remaining roots either lie above the line $L_{1}$ or below the line $L_{2}$. This means that except for $m$ roots, say $\alpha_{1}, \ldots, \alpha_{m}$, all the other roots $\alpha$ satisfy

$$
|\operatorname{Im}(\alpha)| \geq \Delta\left|\sin \left(\frac{2 \pi}{r}\right)\right|
$$

Let $\xi \in \mathbb{R}$. Suppose

$$
d(S, \xi)=\left|\xi-\alpha_{i_{0}}\right|
$$

where $i_{0} \notin\{1, \ldots, m\}$. Then

$$
\begin{aligned}
\left|\xi-\alpha_{1}\right| & \leq\left|\xi-\alpha_{i_{0}}\right|+\left|\alpha_{i_{0}}-\alpha_{1}\right| \\
& \leq\left|\xi-\alpha_{i_{0}}\right|\left(1+\frac{2 M}{\left|\xi-\alpha_{i_{0}}\right|}\right) .
\end{aligned}
$$

Now $\left|\xi-\alpha_{i_{0}}\right| \geq\left|\operatorname{Im}\left(\alpha_{i_{0}}\right)\right| \geq \Delta\left|\sin \left(\frac{2 \pi}{r}\right)\right|$. Thus for $r \gg 1$, we have

$$
\left|\xi-\alpha_{1}\right| \leq\left|\xi-\alpha_{i_{0}}\right|\left(1+\frac{M r}{2 \Delta}\right) \text {. }
$$


Thus

$$
\left|\xi-\alpha_{1}\right| \leq R_{2}\left|\xi-\alpha_{i_{0}}\right| \text {. }
$$

Hence there is a set $S_{2}$ of $m$ roots such that for any real number $\xi$, we have

$$
d\left(S_{2}, \xi\right) \leq R_{2} d(S, \xi)
$$

This completes the proof of the lemma.

\section{Proof of Theorem 1.9}

We begin by stating a lemma of Lewis and Mahler [11, Lemma 1] on Diophantine approximation.

Lemma 4.1. If $(x, y)$ is a solution of (2) with $y \neq 0$ and $H(x, y)=$ $\max (|x|,|y|)>B^{1 / r}$, then

$$
d\left(S, \frac{x}{y}\right) \leq \frac{B}{H(x, y)^{r}}
$$

Combining this lemma with Lemma 3.3, we obtain the following result.

Lemma 4.2. There is a set $S_{2} \subseteq S$ with $\left|S_{2}\right| \ll \sqrt{r(\log r+\log M)}$ such that

$$
d\left(S_{2}, \frac{x}{y}\right) \leq \frac{B R_{2}}{H(x, y)^{r}}
$$

for any solution $(x, y)$ of (2) with $y \neq 0$ and $H(x, y)>B^{1 / r}$.

Let $a, b, \delta, t, \lambda$ and $A$ be as in Section 1.3. Following [3], we say that a rational number $x / y$ is a very good approximation to $\alpha \in S$ if

$$
\left|\alpha-\frac{x}{y}\right|<\left(4 e^{A} H(x, y)\right)^{-\lambda} \text {. }
$$

We now state the Thue-Siegel principle as given in [3, p. 74].

Lemma 4.3. Let $\alpha \in S$. If $x / y, x^{\prime} / y^{\prime}$ are two very good approximations to $\alpha$, then

$$
\log \left(4 e^{A}\right)+\log H\left(x^{\prime}, y^{\prime}\right) \leq \delta^{-1}\left\{\log \left(4 e^{A}\right)+\log H(x, y)\right\} .
$$

\section{Proof of Theorem 1.9}

(i) We argue as in [3] and [8]. Let $\alpha_{i} \in S_{1}$, where $S_{1}$ is given by Lemma 3.1. We first count all large primitive solutions of (2) which are closest to $\alpha_{i}$. Let

$$
I_{i}=\left\{(x, y): y>Y_{G} R_{1}^{\frac{1}{r-2}+\frac{1}{r^{2}}}, \operatorname{gcd}(x, y)=1,\left|\alpha_{i}-\frac{x}{y}\right|=d\left(S_{1}, \frac{x}{y}\right)\right\} .
$$


In Lemma 2.1 we take $U=I_{i}$. Suppose that $\left|I_{i}\right|=n$. Enumerate the elements of $I_{i}$ as $\left(x_{1}, y_{1}\right),\left(x_{2}, y_{2}\right), \ldots,\left(x_{n}, y_{n}\right)$ with $H\left(x_{1}, y_{1}\right) \leq$ $H\left(x_{2}, y_{2}\right) \leq \cdots \leq H\left(x_{n}, y_{n}\right)$. Take $T\left(\left(x_{j}, y_{j}\right)\right)=H\left(x_{j}, y_{j}\right)$. Then

$$
\frac{1}{y_{j} y_{j+1}} \leq\left|\frac{x_{j+1}}{y_{j+1}}-\frac{x_{j}}{y_{j}}\right| \leq\left|\frac{x_{j+1}}{y_{j+1}}-\alpha_{i}\right|+\left|\alpha_{i}-\frac{x_{j}}{y_{j}}\right| \leq \frac{2 B R_{1}}{H\left(x_{j}, y_{j}\right)^{r}}
$$

by Lemmas 3.1 and 4.1 . Thus,

$$
H\left(x_{j+1}, y_{j+1}\right) \geq H\left(x_{j}, y_{j}\right)^{r-1} /\left(2 B R_{1}\right) .
$$

Hence (14) is valid with

$$
\beta=1 /\left(2 B R_{1}\right)<1, \gamma=r-1 .
$$

We first estimate the number $n_{1}$ of elements $(x, y)$ in $I_{i}$ satisfying

$$
H(x, y) \geq\left(\frac{R_{1}}{\sqrt{|D|}}\right)^{\frac{1}{r-\lambda}} Y_{E}=\left(2 B R_{1}\right)^{\frac{1}{r-\lambda}}\left(4 e^{A}\right)^{\frac{\lambda}{r-\lambda}}
$$

All such solutions are very good approximations to $\alpha_{i}$. Hence by Lemma 4.3, we have

$$
\log T\left(\left(x_{n}, y_{n}\right)\right) \leq \delta^{-1}\left(\log \left(4 e^{A}\right)+\log T\left(\left(x_{1}, y_{1}\right)\right)\right)
$$

i.e.

$$
T\left(\left(x_{n}, y_{n}\right)\right) \leq\left(4 e^{A} T\left(\left(x_{1}, y_{1}\right)\right)\right)^{1 / \delta}
$$

In Lemma 2.1(ii), take

$$
\begin{gathered}
A_{1}=\left(2 B R_{1}\right)^{\frac{1}{r-\lambda}}\left(4 e^{A}\right)^{\frac{\lambda}{r-\lambda}}, \\
\eta_{1}=4 e^{A}, \eta_{2}=1 / \delta, \mu=\lambda \text { and } \nu=r-\lambda .
\end{gathered}
$$

As mentioned in Remark [1.2, we may take $r$ sufficiently large. Note that $1 / \delta \sim r$. Hence $1<\eta_{2} \leq \gamma^{n-1}$ for $n \geq 3$. Also, $\mu=\lambda \sim \sqrt{r}$. Hence $1 \leq \mu<\nu=r-\lambda<r-2$. Thus

$$
n_{1} \ll 1+\frac{1}{\log (r-1)} \log \left(r \max \left(\frac{r}{\lambda}, \frac{r-2}{\lambda-2}\right)\right) \ll 1 .
$$

Next, we estimate the number $n_{2}$ of solutions $(x, y)$ satisfying

$$
Y_{G} R_{1}^{\frac{1}{r-2}+\frac{1}{r^{2}}}<H(x, y) \leq\left(\frac{R_{1}}{\sqrt{|D|}}\right)^{1 /(r-\lambda)} Y_{E} \leq\left(2 B R_{1}\right)^{\frac{1+\lambda / a^{2}}{r-\lambda}} .
$$

Applying Lemma 2.1(i) with

$$
\begin{gathered}
A_{1}=Y_{G} R_{1}^{\frac{1}{r-2}+\frac{1}{r^{2}}}=\left(2 B R_{1}\right)^{\frac{1}{r-2}+\frac{1}{r^{2}}}, \\
B_{1}=\left(2 B R_{1}\right)^{\frac{1+\lambda / a^{2}}{r-\lambda}}, \beta=1 /\left(2 B R_{1}\right), \kappa=1 \text { and } \gamma=r-1,
\end{gathered}
$$


we obtain that $n_{2} \ll 1$. Thus

$$
\left|I_{i}\right|=n_{1}+n_{2} \ll 1 \text {. }
$$

As this inequality is true for every root $\alpha_{i} \in S_{1}$ and $\left|S_{1}\right| \ll s$, we obtain that the number of solutions $(x, y)$ with $H(x, y)>Y_{G} R_{1}^{\frac{1}{r-2}+\frac{1}{r^{2}}}$ is $\ll s$.

The proof of $(i i)$ is similar to the above proof with $R_{1}, S_{1}$ replaced by $R_{2}, S_{2}$.

In Sections 5-7, we improve the mechanism for dealing with medium solutions developed by Mueller and Schmidt.

\section{Archimedean NeWton polygon and Large DeRivatives}

Let $F$ be as in (1). The Archimedean Newton polygon of $f(z)=$ $F(z, 1)$ is the lower boundary of the convex hull of the points

$$
P_{i}=\left(r_{i},-\log \left|a_{i}\right|\right), i=0, \ldots, s .
$$

We label the vertices of the Newton polygon as

$$
P_{0}=P_{i(0)}, P_{i(1)}, \ldots, P_{i(\ell)}=P_{s},
$$

where $0=i(0)<i(1)<\ldots<i(\ell)=s$. For $k=1, \ldots, \ell$, define $\sigma(i(k))$ as the slope of the line segment $P_{i(k-1)}, P_{i(k)}$. For $k=0, \ldots, \ell-1$, define $\sigma^{+}(i(k))$ as the slope of the line segment $P_{i(k)}, P_{i(k+1)}$. Let $\alpha$ be a root of $f$. We define $K(\alpha), k(\alpha)$ as follows. If $\sigma^{+}(i(\ell-1))=\sigma(s)<$ $\log |\alpha|+\Psi+\log 3$, then put $K(\alpha)=\ell$. If not, then define $K(\alpha)$ as the least integer $K$ in $0 \leq K \leq \ell-1$ with $\sigma^{+}(i(K)) \geq \log |\alpha|+\Psi+\log 3$. If $\sigma(i(1))=\sigma^{+}(0)>\log |\alpha|-\Psi-\log 3$, then put $k(\alpha)=0$. Otherwise, define $k(\alpha)$ as the largest integer $k$ in $1 \leq k \leq \ell$ with $\sigma(i(k)) \leq$ $\log |\alpha|-\Psi-\log 3$. Clearly, $k(\alpha) \leq K(\alpha)$. In [18], $k(\alpha)$ and $K(\alpha)$ are defined with $\Psi$ replaced by $\log s$. As in [18, Equation (6.3)], we have

$$
k(\alpha)<K(\alpha)
$$

for every root $\alpha$ of $f$.

Lemma 5.1. The coefficients of $F$ satisfy condition (10) if and only if the Newton polygon of $F$ is a straight line joining $\left(0,-\log \left|a_{0}\right|\right)$ and $\left(r,-\log \left|a_{s}\right|\right)$. Further, in this case the height $H$ of $F$ is either $\left|a_{0}\right|$ or $\left|a_{s}\right|$.

Proof. The Newton polygon is a straight line if and only if the slope of the line joining $\left(0,-\log \left|a_{0}\right|\right)$ and $\left(r,-\log \left|a_{s}\right|\right)$ does not exceed 
the slope of the line joining $\left(0,-\log \left|a_{0}\right|\right)$ and $\left(r_{i},-\log \left|a_{i}\right|\right)$ for $i=$ $1, \ldots, s-1$, i.e. for every $i$ with $1 \leq i \leq s-1$, we have

$$
\frac{-\log \left|a_{s}\right|+\log \left|a_{0}\right|}{r} \leq \frac{-\log \left|a_{i}\right|+\log \left|a_{0}\right|}{r_{i}}
$$

or,

$$
\left|\frac{a_{0}}{a_{s}}\right|^{1 / r} \leq\left|\frac{a_{0}}{a_{i}}\right|^{1 / r_{i}}
$$

which is condition (10). This proves the first assertion.

Condition (10) means that for $i=1, \ldots, s-1$, we have

$$
\left|a_{s}\right|\left|a_{0}\right|^{\frac{r}{r_{i}}-1} \geq\left|a_{i}\right|^{\frac{r}{r_{i}}}
$$

If $\left|a_{0}\right| \leq\left|a_{s}\right|$, then the above inequality implies that

$$
\left|a_{s}\right|^{\frac{r}{r_{i}}} \geq\left|a_{i}\right|^{\frac{r}{r_{i}}} \text { for } i=1, \ldots, s-1 \text {. }
$$

Thus

$$
\left|a_{s}\right| \geq\left|a_{i}\right| \text { for } i=0, \ldots, s-1 \text {. }
$$

Hence $H=\left|a_{s}\right|$. Similarly, if $\left|a_{s}\right| \leq\left|a_{0}\right|$, then we get

$$
\left|a_{0}\right|^{\frac{r}{r_{i}}} \geq\left|a_{i}\right|^{\frac{r}{r_{i}}} \text { for } i=1, \ldots, s-1 \text {. }
$$

Thus $H=\left|a_{0}\right|$. This proves the second assertion.

Remark 5.2. By Lemma 5.1, when the coefficients of F satisfy (10), we have $\ell=1$. Since $k(\alpha)<K(\alpha)$, we find

$$
k(\alpha)=0 \text { and } K(\alpha)=1
$$

for any $\alpha \in S$.

As a consequence of Lemma 5.1, we get the following result which is a special case of Lemma $1(i)$ of [18].

Lemma 5.3. Suppose that the coefficients of F satisfy (10). Then for every root $\alpha$ of $f$, we have

$$
\frac{1}{2} e^{\sigma}<|\alpha|<2 e^{\sigma}
$$

where $\sigma$ denotes the slope of the line joining $\left(0,-\log \left|a_{0}\right|\right)$ and $\left(r,-\log \left|a_{s}\right|\right)$.

Proof. By Lemma [5.1, the coefficients of $F$ satisfy (10) if and only if

$$
\sigma \leq \frac{-\log \left|a_{i}\right|+\log \left|a_{0}\right|}{r_{i}} \text { for } i=1, \ldots, s-1 .
$$

This implies that

$$
\left|a_{s}\right| e^{\sigma r}=\left|a_{0}\right| \geq\left|a_{i}\right| e^{\sigma r_{i}} \text { for } i=0, \ldots, s-1 .
$$


When $z=e^{\sigma} w$ with $|w| \geq 2$, we have

$$
\begin{aligned}
|f(z)| & =\left|a_{s} e^{\sigma r} w^{r}+a_{s-1} e^{\sigma r_{s-1}} w^{r_{s-1}}+\cdots+a_{0}\right| \\
& \geq\left|a_{s} e^{\sigma r}\right|\left|w^{r}\right|-\left|a_{s-1} e^{\sigma r_{s-1}}\right|\left|w^{r_{s-1}}\right|-\cdots-\left|a_{0}\right| \\
& \geq\left|a_{s} e^{\sigma r}\right|\left(\left|w^{r}\right|-\left|w^{r_{s-1}}\right|-\cdots-1\right)>0 .
\end{aligned}
$$

Therefore if $\alpha$ is a root of $f$, then

$$
|\alpha|<2 e^{\sigma}
$$

Now consider the reciprocal polynomial $\hat{f}$ of $f$, i.e.

$$
\hat{f}(z)=z^{r} f(1 / z) .
$$

The Newton polygon of $\hat{f}$ is the single line with slope $-\sigma$. Hence every root $\hat{\alpha}$ of $\hat{f}$ satisfies

$$
|\hat{\alpha}|<2 e^{-\sigma}
$$

Since the roots of $\hat{f}$ are the reciprocals of the roots of $f$, we obtain

$$
|\alpha|>\frac{1}{2} e^{\sigma}
$$

for every root $\alpha$ of $f$. This completes the proof of the lemma.

We shall now use the Newton polygon to prove that for each root $\alpha$ of $f$, there exists $u$ with $1 \leq u \leq r$ such that $\left|f^{(u)}(\alpha)\right|$ is large. This will enable us to obtain good rational approximations to $\alpha$ from the solutions of (2) (See Lemma 6.1). We introduce some notation. Let $e, h$ be two non-negative integers. Let $(e)_{h}$ be the Pochhammer symbol defined as

$$
(e)_{h}= \begin{cases}0 & \text { if } e=0 \\ 1 & \text { if } h=0 \\ e(e-1) \cdots(e-h+1) & \text { otherwise }\end{cases}
$$

For a positive integer $t$, define

$$
\Delta_{t}^{-}(e)=\left(\begin{array}{c}
(e)_{0} \\
\vdots \\
(e)_{t}
\end{array}\right)
$$


Further, for $0 \leq u \leq t$, let

$$
\Delta_{t, u}^{-}(e)=\left(\begin{array}{c}
(e)_{0} \\
\vdots \\
(e)_{u-1} \\
(e)_{u+1} \\
\vdots \\
(e)_{t}
\end{array}\right) .
$$

If $\left\{a_{1}, \ldots, a_{t+1}\right\}$ is a set of positive integers, then

$$
\operatorname{det}\left(\Delta_{t}^{-}\left(a_{1}\right), \ldots, \Delta_{t}^{-}\left(a_{t+1}\right)\right)=\prod_{1 \leq i<j \leq t+1}\left(a_{j}-a_{i}\right) .
$$

Let $\left\{b_{1}, \ldots, b_{t}\right\}$ be any set of positive integers. Put

$$
E_{u}^{(t)}=E_{u}^{(t)}\left(b_{1}, \ldots, b_{t}\right)=(-1)^{t+u} \operatorname{det}\left(\Delta_{t, u}^{-}\left(b_{1}\right), \ldots, \Delta_{t, u}^{-}\left(b_{t}\right)\right) .
$$

Then for any positive integer $e$, we have

$$
\sum_{u=0}^{t}(e)_{u} E_{u}^{(t)}\left(b_{1}, \ldots, b_{t}\right)=\operatorname{det}\left(\Delta_{t}^{-}\left(b_{1}\right), \ldots, \Delta_{t}^{-}\left(b_{t}\right), \Delta_{t}^{-}(e)\right) .
$$

We denote $\operatorname{det}\left(\Delta_{t}^{-}\left(b_{1}\right), \ldots, \Delta_{t}^{-}\left(b_{t}\right), \Delta_{t}^{-}(e)\right)$ by $D\left(b_{1}, \ldots, b_{t}, e\right)$. Note that $D\left(b_{1}, \ldots, b_{t}, e\right)=0$ whenever $e=b_{i}$ for any $i$ with $1 \leq i \leq t$.

Lemma 5.4. Let $P(z)=p_{1} z^{e_{1}}+\ldots+p_{m} z^{e_{m}}, e_{1}<\ldots<e_{m}$, be a polynomial and let $\left\{b_{1}, \ldots, b_{t}\right\}$ be any set of positive integers. Then

$$
\sum_{u=0}^{t} E_{u}^{(t)} z^{u} P^{(u)}(z)=\sum_{i=1}^{m} p_{i} z^{e_{i}} D\left(b_{1}, \ldots, b_{t}, e_{i}\right),
$$

where $E_{u}^{(t)}=E_{u}^{(t)}\left(b_{1}, \ldots, b_{t}\right)$.

Proof. Observe that for $0 \leq u \leq t$, we have

$$
z^{u} P^{(u)}(z)=\left(e_{1}\right)_{u} p_{1} z^{e_{1}}+\ldots+\left(e_{m}\right)_{u} p_{m} z^{e_{m}} .
$$

Then

$$
\begin{aligned}
\sum_{u=0}^{t} E_{u}^{(t)} z^{u} P^{(u)}(z) & =p_{1} z^{e_{1}} \sum_{u=0}^{t}\left(e_{1}\right)_{u} E_{u}^{(t)}+\ldots+p_{m} z^{e_{m}} \sum_{u=0}^{t}\left(e_{m}\right)_{u} E_{u}^{(t)} \\
& =p_{1} z^{e_{1}} D\left(b_{1}, \ldots, b_{t}, e_{1}\right)+\ldots+p_{m} z^{e_{m}} D\left(b_{1}, \ldots, b_{t}, e_{m}\right) .
\end{aligned}
$$

This proves the lemma. 
Corollary 5.5. Let $\alpha$ be a root of $f(z)$. Then

$$
\sum_{u=1}^{i(K)} E_{u}^{(i(K))} \alpha^{u} f^{(u)}(\alpha)=a_{i(K)} D_{i(K)}^{(1)} \alpha^{r_{i(K)}}+\sum_{j>i(K)} a_{j} D_{j}^{(1)} \alpha^{r_{j}},
$$

where $K=K(\alpha), E_{u}^{(i(K))}=E_{u}^{(i(K))}\left(r_{0}, \ldots, r_{i(K)-1}\right)$, $D_{j}^{(1)}=D\left(r_{0}, \ldots, r_{i(K)-1}, r_{j}\right)$ and the sum with $j>i(K)$ is taken as zero if $K=\ell$. Also

$$
\sum_{u=1}^{s-i(k)} E_{u}^{(s-i(k))} \alpha^{u} f^{(u)}(\alpha)=a_{i(k)} D_{i(k)}^{(2)} \alpha^{r_{i(k)}}+\sum_{j<i(k)} a_{j} D_{j}^{(2)} \alpha^{r_{j}}
$$

where $k=k(\alpha), E_{u}^{(s-i(k))}=E_{u}^{(s-i(k))}\left(r_{i(k)+1}, \ldots, r_{s}\right)$, $D_{j}^{(2)}=D\left(r_{i(k)+1}, \ldots, r_{s}, r_{j}\right)$ and the sum with $j<i(k)$ is taken as zero if $k=0$.

Proof. In Lemma 5.4, take $P(z)=f(z)$ with $\left(e_{1}, \ldots, e_{m}\right)=\left(r_{0}, \ldots, r_{s}\right)$ and $\left(p_{1}, \ldots, p_{m}\right)=\left(a_{0}, \ldots, a_{s}\right)$. Now (20) follows from the lemma by taking $z=\alpha, t=i(K),\left(b_{1}, \ldots, b_{t}\right)=\left(r_{0}, \ldots, r_{i(K)-1}\right)$ and using the fact that $D\left(r_{0}, \ldots, r_{i(K)-1}, r_{j}\right)=0$ for $j<i(K)$. Similarly, we obtain (21) by taking $z=\alpha, t=s-i(k),\left(b_{1}, \ldots, b_{t}\right)=\left(r_{i(k)+1}, \ldots, r_{s}\right)$ and using the fact that $D\left(r_{i(k)+1}, \ldots, r_{s}, r_{j}\right)=0$ for $j>i(k)$.

Note that the sums on the left hand sides of (20) and (21) are nonempty since $0 \leq i(k)<i(K) \leq s$.

Lemma 5.6. Let $(x, y)$ be a solution of (2) with $y \neq 0$. Let $\alpha$ be a root of $f$. Then

(i) There exists $u$ with $1 \leq u \leq i(K)$ such that

$$
\left|f^{(u)}(\alpha)\right| \geq \frac{1}{4 s}\left(2 s^{2} r\right)^{1-s}\left|a_{i(K)}\right||\alpha|^{r_{i(K)}-u} .
$$

(ii) There exists $v$ with $1 \leq v \leq s-i(k)$ such that

$$
\left|f^{(v)}(\alpha)\right| \geq \frac{1}{4 s}\left(2 s^{2} r\right)^{1-s}\left|a_{i(k)}\right||\alpha|^{r_{i(k)}-v} .
$$

Proof. Suppose that $K<\ell$. Let $j>i(K)$. Put

$$
W_{j}^{(1)}=\left|\frac{a_{j} D_{j}^{(1)} \alpha^{r_{j}}}{a_{i(K)} D_{i(K)}^{(1)} \alpha^{r_{i(K)}}}\right|
$$


By (20), we have

$$
\sum_{u=1}^{i(K)} E_{u}^{(i(K))} \alpha^{u} f^{(u)}(\alpha)=a_{i(K)} D_{i(K)}^{(1)} \alpha^{r_{i(K)}}\left(1+\sum_{j>i(K)} \frac{a_{j} D_{j}^{(1)} \alpha^{r_{j}}}{a_{i(K)} D_{i(K)}^{(1)} \alpha^{r_{i(K)}}}\right) .
$$

From the definition of $D_{j}^{(1)}$ and (19) , we obtain

$$
\left|\frac{D_{j}^{(1)}}{D_{i(K)}^{(1)}}\right|=\prod_{w<i(K)}\left(\frac{r_{j}-r_{w}}{r_{i(K)}-r_{w}}\right)=\prod_{w<i(K)}\left(1+\left(\frac{r_{j}-r_{i(K)}}{r_{i(K)}-r_{w}}\right)\right) .
$$

This implies

$$
\begin{aligned}
\log \left|\frac{D_{j}^{(1)}}{D_{i(K)}^{(1)}}\right| & \leq\left(r_{j}-r_{i(K)}\right) \sum_{w<i(K)} \frac{1}{r_{i(K)}-r_{w}} \\
& \leq\left(r_{j}-r_{i(K)}\right) \Psi .
\end{aligned}
$$

Then

$$
\begin{aligned}
\log W_{j}^{(1)} & \leq\left(r_{j}-r_{i(K)}\right)(\log |\alpha|+\Psi)+\log \left|a_{j}\right|-\log \left|a_{i(K)}\right| \\
& =\left(r_{j}-r_{i(K)}\right)(\log |\alpha|+\Psi-\sigma(j, i(K))),
\end{aligned}
$$

where $\sigma(j, i(K))$ denotes the slope of the line segment joining $P_{i(K)}$ and $P_{j}$. From the convexity of the Newton polygon and the definition of $K=K(\alpha)$, we get

$$
\sigma(j, i(K)) \geq \sigma^{+}(i(K)) \geq \log |\alpha|+\Psi+\log 3 .
$$

Hence

$$
\log W_{j}^{(1)} \leq-\left(r_{j}-r_{i(K)}\right) \log 3
$$

implying that

$$
W_{j}^{(1)} \leq 3^{-\left(r_{j}-r_{i(K)}\right)} .
$$

Thus

$$
\sum_{j>i(K)} W_{j}^{(1)} \leq 3^{-1}+3^{-2}+\ldots=\frac{1}{2} .
$$

Using this in (22), we obtain

$$
\left|\sum_{u=1}^{i(K)} E_{u}^{(i(K))} \alpha^{u} f^{(u)}(\alpha)\right| \geq \frac{1}{2}\left|a_{i(K)} D_{i(K)}^{(1)} \alpha^{r_{i(K)}}\right| .
$$


It is easy to see that the above inequality holds also for $K=\ell$ since then the right hand side of (22) reduces to $\left|a_{s} D_{s}^{(1)} \alpha^{r}\right|$. Suppose that $k>0$. As above, it can be shown that for $j<i(k)$,

$$
\log \left|\frac{D_{j}^{(2)}}{D_{i(k)}^{(2)}}\right| \leq\left(r_{i(k)}-r_{j}\right) \Psi
$$

and hence

$$
\left|\sum_{u=1}^{s-i(k)} E_{u}^{(s-i(k))} \alpha^{u} f^{(u)}(\alpha)\right| \geq \frac{1}{2}\left|a_{i(k)} D_{i(k)}^{(2)} \alpha^{r_{i(k)}}\right| .
$$

This inequality is also true for $k=0$. From [18, Eqns (6.12) \& (6.13)] it follows that

$$
\left|E_{u}^{(i(K))}\right| \leq 2^{s}\left(s^{2} r\right)^{s-1}\left|D_{i(K)}^{(1)}\right| \text { for } 1 \leq u \leq i(K)
$$

and

$$
\left|E_{u}^{(s-i(k))}\right| \leq 2^{s}\left(s^{2} r\right)^{s-1}\left|D_{i(k)}^{(2)}\right| \text { for } 1 \leq u \leq s-i(k) .
$$

Substituting (25) in (23), we find that

$$
\left|f^{(u)}(\alpha)\right| \geq \frac{1}{4 s}\left(2 s^{2} r\right)^{1-s}\left|a_{i(K)}\right||\alpha|^{r_{i(K)}-u}
$$

for some $u$ with $1 \leq u \leq i(K)$. In a similar manner, (24) and (26) yield the second part of the lemma.

\section{Good Rational approximations}

The results in this section correspond to [18, Section 14], with minor changes. We include the details for the convenience of the reader. Throughout this section, assume that $(x, y)$ is a solution of (2) with $y \neq 0$ and that $\alpha$ is a root of $f$ with

$$
d\left(S, \frac{x}{y}\right)=\left|\alpha-\frac{x}{y}\right| .
$$

Let $q$ be the smallest integer with

$$
\left|a_{q}\right|=H=\max _{0 \leq j \leq s}\left|a_{j}\right| .
$$

Note that $\left(q,-\log \left|a_{q}\right|\right)$ is a vertex of the Newton polygon of $f$. The following lemma from [18] gives a rational approximation to $\alpha$ in terms of the derivatives of $f$. 
Lemma 6.1. [18, Lemma 10] If $f^{(u)}(\alpha) \neq 0$ for some $u$ in $1 \leq u \leq r$, then

$$
d\left(S, \frac{x}{y}\right) \leq \frac{r}{2}\left(\frac{2^{r} h}{\left|f^{(u)}(\alpha) y^{r}\right|}\right)^{1 / u} .
$$

The following two results are a consequence of the above lemma.

Lemma 6.2. If $q<i(K)$, where $K=K(\alpha)$, then

$$
d\left(S, \frac{x}{y}\right) \leq \frac{1}{H^{(1 / u)-(1 / r)}}\left(\frac{(r s)^{2 s}\left(6 e^{\Psi}\right)^{r} h}{|y|^{r}}\right)^{1 / u}
$$

where $u$ is chosen according to Lemma 5.6 $(i)$.

Proof. Combining Lemmas 5.6(i) and 6.1, we obtain

$$
\left|\alpha-\frac{x}{y}\right| \leq\left(\frac{2^{r}(r s)^{2 s} h}{\left|a_{i(K)}\right||\alpha|^{r_{i(K)}-u}|y|^{r}}\right)^{1 / u} .
$$

Denote $\left|a_{i(K)}\right||\alpha|^{r_{i(K)}-u}$ by $\Delta(\alpha, u)$. Thus

$$
\log \Delta(\alpha, u)=\left(r_{i(K)}-u\right) \log |\alpha|+\log \left|a_{i(K)}\right| .
$$

From the definition of $K(\alpha)$ it follows that

$$
\log |\alpha| \geq \sigma(i(K))-\Psi-\log 3 .
$$

Since $q<i(K)$, we have $\sigma(i(K)) \geq \sigma(q, i(K))$ as the Newton polygon is convex. Thus

$$
\begin{aligned}
\log \Delta(\alpha, u) & \geq\left(r_{i(K)}-u\right)(\sigma(i(K))-\Psi-\log 3)+\log \left|a_{i(K)}\right| \\
& \geq\left(r_{i(K)}-u\right) \sigma(q, i(K))+\log \left|a_{i(K)}\right|-r \log \left(3 e^{\Psi}\right) \\
& =\left(r_{q}-u\right) \sigma(q, i(K))+\log \left|a_{q}\right|-r \log \left(3 e^{\Psi}\right) .
\end{aligned}
$$

Since $P_{q}$ is one of the lowest vertices of the Newton polygon and $q<$ $i(K)$, the slope $\sigma(q, i(K))$ is non-negative. Therefore if $r_{q} \geq u$, we have

$$
\log \Delta(\alpha, u) \geq \log \left|a_{q}\right|-r \log \left(3 e^{\Psi}\right)=\log H-r \log \left(3 e^{\Psi}\right) .
$$

If $r_{q}<u$, we have $\sigma(q, i(K)) \leq \sigma(q, s)$. Hence

$$
\begin{aligned}
\log \Delta(\alpha, u) & \geq\left(r_{q}-u\right) \sigma(q, s)+\log \left|a_{q}\right|-r \log \left(3 e^{\Psi}\right) \\
& =\left(1-\frac{u-r_{q}}{r_{s}-r_{q}}\right) \log \left|a_{q}\right|+\frac{u-r_{q}}{r_{s}-r_{q}} \log \left|a_{s}\right|-r \log \left(3 e^{\Psi}\right) \\
& \geq\left(1-\frac{u-r_{q}}{r-r_{q}}\right) \log \left|a_{q}\right|-r \log \left(3 e^{\Psi}\right) \\
& \geq\left(1-\frac{u}{r}\right) \log H-r \log \left(3 e^{\Psi}\right) .
\end{aligned}
$$


Substituting this in (28), we obtain the assertion of the lemma.

Lemma 6.3. If $x \neq 0,|y| \geq 2(r s)^{2 s / r} h^{1 / r}$ and $i(k)<q$, where $k=$ $k(\alpha)$, then

$$
d\left(S^{*}, \frac{y}{x}\right) \leq \frac{1}{H^{(1 / v)-(1 / r)}}\left(\frac{(r s)^{2 s}\left(12 e^{\Psi}\right)^{r} h}{|x|^{r}}\right)^{1 / v}
$$

where $v$ is chosen according to Lemma 5.6(ii) and $S^{*}=\left\{\alpha^{-1} \mid \alpha \in S\right\}$.

Proof. Combining Lemmas 5.6(ii) and 6.1, we obtain

$$
\left|\alpha-\frac{x}{y}\right| \leq\left(\frac{2^{r}(r s)^{2 s} h}{\left|a_{i(k)}\right||\alpha|^{r_{i(k)}-v}|y|^{r}}\right)^{1 / v} .
$$

Denote $\left|a_{i(k)}\right||\alpha|^{r_{i(k)}-v}$ by $\Delta^{*}(\alpha, v)$. Then

$$
\log \left(|\alpha|^{v} \Delta^{*}(\alpha, v)\right)=r_{i(k)} \log |\alpha|+\log \left|a_{i(k)}\right| .
$$

If $k=0$, we have $r_{i(0)}=0$ and hence $\log \left(|\alpha|^{v} \Delta^{*}(\alpha, v)\right) \geq 0$. Now suppose that $k>0$. From the definition of $k(\alpha)$ it follows that

$$
\log |\alpha| \geq \sigma(i(k))+\Psi+\log 3
$$

This implies that

$$
\begin{aligned}
\log \left(|\alpha|^{v} \Delta^{*}(\alpha, v)\right) & \geq r_{i(k)} \sigma(i(k))+\log \left|a_{i(k)}\right| \\
& \geq r_{i(k)} \sigma(0, i(k))+\log \left|a_{i(k)}\right|=\log \left|a_{0}\right| \geq 0 .
\end{aligned}
$$

Therefore

$$
\left|\alpha-\frac{x}{y}\right| \leq|\alpha|\left(\frac{2^{r}(r s)^{2 s} h}{|y|^{r}}\right)^{1 / v} \leq|\alpha|
$$

by the assumption on $y$. Hence

$$
|x| \leq|2 \alpha y|
$$

Using this we obtain

$$
\begin{aligned}
\left|\alpha^{-1}-\frac{y}{x}\right| & =\left|\frac{y}{x \alpha}\right|\left|\alpha-\frac{x}{y}\right| \leq\left(\frac{2^{r}(r s)^{2 s} h}{\left|a_{i(k)}\right||\alpha|^{r_{i(k)}}}\right)^{1 / v} \frac{1}{|x||y|^{(r / v)-1}} \\
& \leq\left(\frac{4^{r}(r s)^{2 s} h}{\Gamma(\alpha, v)|x|^{r}}\right)^{1 / v},
\end{aligned}
$$

where $\Gamma(\alpha, v)=\left|a_{i(k)}\right||\alpha|^{-\left(r-r_{i(k)}-v\right)}$. Note that $r-r_{i(k)}-v \geq 0$. It is enough to show that

$$
\Gamma(\alpha, v) \geq\left(3 e^{\Psi}\right)^{-r} H^{1-(v / r)} .
$$


Since $i(k)<q$, we have $\sigma(i(k), q) \geq \sigma^{+}(i(k))>\log |\alpha|-\Psi-\log 3$. Thus

$$
\begin{aligned}
\log \Gamma(\alpha, v) & \geq\left(r-r_{i(k)}-v\right)\left(-\sigma^{+}(i(k))-\log \left(3 e^{\Psi}\right)\right)+\log \left|a_{i(k)}\right| \\
& \geq-\left(r-r_{i(k)}-v\right) \sigma(i(k), q)+\log \left|a_{i(k)}\right|-r \log \left(3 e^{\Psi}\right) \\
& =-\left(r-r_{q}-v\right) \sigma(i(k), q)+\log \left|a_{q}\right|-r \log \left(3 e^{\Psi}\right) .
\end{aligned}
$$

When $v \leq r-r_{q}$, we have

$$
\log \Gamma(\alpha, v) \geq \log \left|a_{q}\right|-r \log \left(3 e^{\Psi}\right)=\log H-r \log \left(3 e^{\Psi}\right)
$$

as $\sigma(i(k), q) \leq 0$. When $v>r-r_{q}$, we get

$$
\begin{aligned}
\log \Gamma(\alpha, v) & \geq-\left(r-r_{q}-v\right) \sigma(0, q)+\log \left|a_{q}\right|-r \log \left(3 e^{\Psi}\right) \\
& =\left(r-r_{q}-v\right)\left(\left(\log \left|a_{q}\right|-\log \left|a_{0}\right|\right) / r_{q}\right)+\log \left|a_{q}\right|-r \log \left(3 e^{\Psi}\right) \\
& \geq\left((r-v) / r_{q}\right) \log \left|a_{q}\right|-r \log \left(3 e^{\Psi}\right) \\
& \geq(1-(v / r)) \log H-r \log \left(3 e^{\Psi}\right) .
\end{aligned}
$$

This proves the claim and hence the assertion of the lemma.

We combine Lemmas 6.2, 6.3 and 3.1 to get the following result, which is analogous to [18, Lemma 17].

Lemma 6.4. There is a set $S_{1}$ of roots of $F(x, 1)$ and a set $S_{1}^{*}$ of roots of $F(1, y)$, both with cardinalities $\leq 6 s+4$, such that any solution $(x, y)$ of (2) with

$$
\min (|x|,|y|) \geq 12 e^{\Psi}(r s)^{2 s / r} h^{1 / r}
$$

either has

$$
\left|\alpha-\frac{x}{y}\right| \leq \frac{R_{1}}{H^{\frac{1}{s}-\frac{1}{r}}}\left(\frac{(r s)^{2 s}\left(12 e^{\Psi}\right)^{r} h}{|y|^{r}}\right)^{1 / s}
$$

for some $\alpha \in S_{1}$ or has

$$
\left|\alpha^{*}-\frac{y}{x}\right| \leq \frac{R_{1}}{H^{\frac{1}{s}-\frac{1}{r}}}\left(\frac{(r s)^{2 s}\left(12 e^{\Psi}\right)^{r} h}{|x|^{r}}\right)^{1 / s}
$$

for some $\alpha^{*} \in S_{1}^{*}$.

Proof. Since $\min (|x|,|y|) \geq 12 e^{\Psi}(r s)^{2 s / r} h^{1 / r}$, Lemmas 6.2 and 6.3 imply that there is either a root $\alpha$ of $F(Z, 1)$ with (27) or a root $\alpha^{-1}$ of $F(1, Z)$ with (29). Further, the right hand sides of these inequalities increase with $u$ and $v$ respectively. Therefore we may replace $u, v$ with $s$. This, together with Lemma 3.1, gives the assertion of the lemma. 


\section{Estimation of Medium SOLUTiOns}

Let $Y_{W}$ be as given in (12). In this section, we shall estimate $P_{\text {med }}\left(Y_{W}, Y\right)$ for suitably chosen $Y$.

Lemma 7.1. Let $F(X, Y)$ be given by (11).

(i) Let

$$
Y_{S}=\left(\left(12 e^{\Psi}\right)^{r} R_{1}^{2 s} h\right)^{\frac{1}{r-2 s}}
$$

Then

$$
P_{\text {med }}\left(Y_{W}, Y_{S}\right) \ll \frac{s}{\Phi}\left(\log s+\log \left(1+\log h^{1 / r}\right)\right) .
$$

(ii) Suppose that the coefficients of $F(X, Y)$ satisfy (10) and that $r \geq 4 s$. Let

$$
Y_{S}^{\prime}=\left(8^{r} R_{1}^{s}\left(s^{2} r\right)^{3 s} h\right)^{\frac{1}{r-2 s}}
$$

Then

$$
P_{\text {med }}\left(Y_{W}, Y_{S}^{\prime}\right) \ll s\left(\log s+\log \left(1+\log h^{1 / r}\right)\right) .
$$

Proof. ( $i$ ) By Lemma 6.4, it is enough to estimate the number of primitive pairs $(x, y)$ satisfying (30) for some $\alpha \in S_{1}$ or (31) for some $\alpha^{*} \in S_{1}^{*}$ with

$$
Y_{S} \leq y \leq Y_{W}
$$

We consider the case when (30) is satisfied. The other case is similar. Let $\alpha \in S_{1}$ and let $U=\left\{\left(x_{1}, y_{1}\right), \ldots,\left(x_{\nu}, y_{\nu}\right)\right\}$ be the set of all solutions of (30) with $\operatorname{gcd}\left(x_{i}, y_{i}\right)=1$ and

$$
Y_{S} \leq y_{1} \leq \ldots \leq y_{\nu} \leq Y_{W}
$$

Suppose that $\nu \geq 2$. Then

$$
\begin{aligned}
\frac{1}{y_{i} y_{i+1}} & \leq\left|\frac{x_{i}}{y_{i}}-\frac{x_{i+1}}{y_{i+1}}\right| \leq\left|\alpha-\frac{x_{i}}{y_{i}}\right|+\left|\alpha-\frac{x_{i+1}}{y_{i+1}}\right| \\
& \leq \frac{K_{1}}{2 y_{i}^{r / s}}+\frac{K_{1}}{2 y_{i+1}^{r / s}} \leq \frac{K_{1}}{y_{i}^{r / s}}
\end{aligned}
$$

where

$$
K_{1}=2 R_{1}(r s)^{2}\left(12 e^{\Psi}\right)^{r / s} h^{1 / s} H^{(1 / r)-(1 / s)} .
$$

Thus we have

$$
y_{i+1} \geq K_{1}^{-1} y_{i}^{(r / s)-1} .
$$

In Lemma 2.1(i) take $T\left(\left(x_{i}, y_{i}\right)\right)=y_{i}, \beta=\frac{1}{K_{1}}, \gamma=\frac{r-s}{s}, A_{1}=Y_{S}$ and $B_{1}=Y_{W}$. Note that $\gamma=(r / s)-1>4 e^{2 \Phi}-1 \geq e^{\Phi} \geq 2$. Further, since 
$R_{1} \geq 4(r s)^{4}$, we have

$$
\frac{Y_{S}}{K_{1}^{\frac{1}{\kappa(\gamma-1)}}} \geq \frac{R_{1}^{\frac{s}{r-2 s}} H^{\frac{r-s}{2 r(r-2 s)}}}{(r s)^{\frac{2 s}{r-2 s}} 2^{\frac{s}{r-2 s}}} \geq R_{1}^{\frac{s}{2(r-2 s)}} H^{\frac{r-s}{2 r(r-2 s)}} .
$$

Using the inequality $M \leq(r+1) H([12$, Eqn (6)]), we get

$$
\log Y_{W} \ll \sqrt{r}+\log H+\log h^{1 / r} .
$$

Now we apply Lemma 2.1(i) to obtain

$$
\begin{aligned}
\nu & \ll 1+\frac{1}{\log \gamma} \log \left(\frac{2 r(r-2 s)\left(\sqrt{r}+\log H+\log h^{1 / r}\right)}{(r-s) \log H+r s \log R_{1}}\right) \\
& \ll \frac{\log r+\log \left(1+\log h^{1 / r}\right)}{\log \gamma} .
\end{aligned}
$$

If $r \ll s^{3}$, we get

$$
\nu \ll \frac{\log s+\log \left(1+\log h^{1 / r}\right)}{\Phi} .
$$

If $r \gg s^{3}$, then $\gamma \gg r^{2 / 3}$, which implies that

$$
\nu \ll 1+\frac{\log \left(1+\log h^{1 / r}\right)}{\log r} \ll \frac{\log s+\log \left(1+\log h^{1 / r}\right)}{\Phi}
$$

as $\Phi \ll \log s$ (see Remark 1.3). Since this is true for each $\alpha \in S_{1}$, we obtain the assertion of part $(i)$ of the lemma.

(ii) By Remark 5.2 and Lemmas 6.1, 5.6 and 3.1, there is a set $S_{1} \subseteq S$ with $\left|S_{1}\right| \ll s$, such that for some $\alpha \in S_{1}$,

$$
d\left(S_{1}, \frac{x}{y}\right)=\left|\alpha-\frac{x}{y}\right| \leq \frac{r R_{1}}{2}\left(\frac{s\left(r s^{2}\right)^{s-1} 2^{r+s+1} h}{|y|^{r}\left|a_{s}\right||\alpha|^{r-u}}\right)^{1 / u} .
$$

By Lemma 5.3, it follows that every root $\alpha$ of $f$ satisfies

$$
\frac{1}{2} e^{\sigma}<|\alpha|<2 e^{\sigma}
$$

where $\sigma$ is the slope of the line joining $\left(0,-\log \left|a_{0}\right|\right)$ and $\left(r,-\log \left|a_{s}\right|\right)$. This implies that

$$
\log |\alpha|>\sigma-\log 2=\frac{-\log \left|a_{s}\right|+\log \left|a_{0}\right|}{r}-\log 2 .
$$

Therefore

$$
\left|a_{s}\right||\alpha|^{r-u} \geq \frac{\left|a_{s}\right|^{u / r}\left|a_{0}\right|^{(r-u) / r}}{2^{r-u}} .
$$


By (32) and $|y| \geq Y_{S}^{\prime}$, we get that

$$
d\left(S_{1}, \frac{x}{y}\right) \leq \frac{r R_{1}}{2}\left(\frac{s\left(r s^{2}\right)^{s-1} 2^{3 r} h}{|y|^{r}\left|a_{s}\right|^{s / r}\left|a_{0}\right|^{(r-s) / r}}\right)^{1 / s} .
$$

As the height of $F$ is either $\left|a_{0}\right|$ or $\left|a_{s}\right|$, we obtain that

$$
\left|\alpha-\frac{x}{y}\right| \leq \frac{r R_{1}}{2 H^{1 / r}}\left(\frac{s\left(r s^{2}\right)^{s-1} 2^{3 r} h}{|y|^{r}}\right)^{1 / s} .
$$

Let $U=\left\{\left(x_{1}, y_{1}\right), \ldots,\left(x_{\nu}, y_{\nu}\right)\right\}$ be the set of all the solutions of (33) with $\operatorname{gcd}\left(x_{i}, y_{i}\right)=1$ and

$$
Y_{S}^{\prime} \leq y_{1} \leq \ldots \leq y_{\nu} \leq Y_{W}
$$

Put

$$
K_{2}=\frac{R_{1} 8^{r / s}(s h)^{1 / s}(r s)^{2}}{H^{1 / r}}
$$

Suppose that $\nu \geq 2$. As in the proof of $(i)$, we get from (33) that

$$
y_{i+1} \geq K_{2}^{-1} y_{i}^{(r / s)-1} \text {. }
$$

In Lemma 2.1(i) take $T\left(\left(x_{i}, y_{i}\right)\right)=y_{i}, \beta=\frac{1}{K_{2}}, \gamma=\frac{r-s}{s}, A_{1}=Y_{S}^{\prime}$ and $B_{1}=Y_{W}$. Note that

$$
Y_{S}^{\prime} \geq K_{2}^{\frac{1}{\kappa(\gamma-1)}} H^{\frac{s}{2 r(r-2 s)}}\left(r s^{3}\right)^{\frac{s}{r-2 s}} \text { and } \gamma \geq 2 .
$$

Thus by Lemma 2.1(i), we obtain

$$
\begin{aligned}
\nu & \ll 1+\frac{1}{\log \gamma} \log \left(\frac{2 r(r-2 s)\left(\sqrt{r}+\log H+\log h^{1 / r}\right)}{s \log H+2 r s \log \left(r s^{3}\right)}\right) \\
& \ll \frac{\log r+\log \left(1+\log h^{1 / r}\right)}{\log \gamma} .
\end{aligned}
$$

Now we argue as in the proof of $(i)$ to get the assertion.

\section{Estimation of SMALL SOLUTiOnS}

To estimate the number of small solutions, we use the following lemma from [18].

Lemma 8.1. [18, Lemma 18]

Let $F(X, Y)$ be given by (11) and let $r \geq 4 s$. Then for any $Y \geq 1$, we have

$$
P_{s m a}(Y) \ll\left(r s^{2}\right)^{2 s / r} h^{2 / r}+s Y .
$$

Lemma 8.2. Let $F(X, Y)$ be given by (1). Then

$$
P_{s m a}\left(Y_{S}\right) \ll e^{c_{7}(\log s) e^{-2 \Phi}} h^{\frac{2}{r}}+s e^{\Phi+c_{8}\left(\log ^{3} s\right) e^{-\Phi}} h^{\frac{1}{r-2 s}} .
$$


(ii)

$$
P_{\text {sma }}\left(Y_{S}^{\prime}\right) \ll s h^{2 / r} \text { whenever } r \geq s \log ^{3} s .
$$

Proof. ( $i$ ) follows from (9) and Lemma 8.1 with $Y$ as $Y_{S}$. Similarly, (ii) follows by taking $Y$ as $Y_{S}^{\prime}$ in Lemma 8.1 and using $r \geq s \log ^{3} s$.

\section{Proofs of Proposition [1.8 And Theorems 1.1, 1.5}

\section{Proof of Proposition 1.8}

Suppose that $r \geq 4 s$. Let $P(h)$ denote the number of primitive solutions of (2). Then

$$
P(h)=P_{\ell a r}\left(Y_{W}\right)+P_{\text {med }}\left(Y_{W}, Y_{S}\right)+P_{\text {sma }}\left(Y_{S}\right) .
$$

The upper bounds for the three quantities on the right hand side are obtained from (11), Lemma 7.1(i) and Lemma 8.2(i). This together with $\Phi \ll \log s$ (see Remark 1.3) yields

$$
P(h) \ll \rho h^{2 / r},
$$

where

$$
\rho=\frac{s \log s}{\Phi}+s^{c_{7} e^{-2 \Phi}}+s e^{\Phi+c_{8}\left(\log ^{3} s\right) e^{-\Phi}} .
$$

Using a partial summation argument, it was shown in [18, p. 212] that

$$
N_{F}(h) \ll P(h)+h^{1 / r} r^{-1} \sum_{n=1}^{h-1} P(n) n^{-1-(1 / r)} .
$$

Substituting our estimate for $P(h)$, we obtain that

$$
N_{F}(h) \ll \rho h^{2 / r}
$$

When $r<4 s$, we use (3) to obtain

$$
N_{F}(h) \ll s C_{1}(r, h) .
$$

This proves the proposition.

\section{Proof of Theorem 1.1}

Observe that $\Phi \geq 3 \log \log s$. Thus from Proposition 1.8, we get

$$
N_{F}(h) \ll s\left(\log s+e^{\Phi}\right) C_{1}(r, h) \ll s e^{\Phi} C_{1}(r, h) .
$$

\section{Proof of Theorem 1.5}

We have

$$
P(h)=P_{\ell a r}\left(Y_{W}\right)+P_{m e d}\left(Y_{W}, Y_{S}^{\prime}\right)+P_{s m a}\left(Y_{S}^{\prime}\right) .
$$

We use the respective upper bounds for the three quantities on the right hand side from (11), Lemma 7.1(ii) and Lemma 8.2(ii) and proceed as in the proof of Proposition 1.8 to give the assertion. 


\section{REFERENCES}

[1] T. M. Apostol, Introduction to Analytic Number Theory, Springer-Verlag, New York-Heidelberg (1976).

[2] M. Bean, An isoperimetric inequality for the area of plane regions defined by binary forms, Compositio Math. 92 (1994), 115-131.

[3] E. Bombieri and W. M. Schmidt, On Thue's equation, Invent. Math. 88 (1987), 69-81.

[4] P. Borwein and T. Erdélyi, On the zeros of polynomials with restricted coefficients, Illinois J. Math. 41 (1997), no. 4, 667-675.

[5] B. Brindza, Á. Pintér, A. J. van der Poorten and M. Waldschmidt, On the distribution of solutions of Thue's equation, Number theory in progress. 1 (Zakopane-Kościelisko, 1997) de Gruyter, Berlin (1999), 35-46.

[6] J.-H. Evertse, On the equation $a x^{n}-b y^{n}=c$, Compositio Math. 47 (1982), 288-315.

[7] J.-H. Evertse and K. Györy, Thue inequalities with a small number of solutions, The mathematical heritage of C. F. Gauss, World Scientific Publ. Co., Singapore (1991), 204-224.

[8] K. Győry, Thue inequalities with a small number of primitive solutions, Periodica Math. Hungar. 42 (2001), 199-209.

[9] K. Györy, On the number of primitive solutions of Thue equations and Thue inequalities, Paul Erdős and his Mathematics I, Bolyai Soc. Math. Studies, 11 (2002), 279-294.

[10] S. Hyyrö, Über die Gleichung $a x^{n}-b y^{n}=c$ und das Catalansche Problem, Ann. Acad. Sci Fenn., Ser. AI 355 (1964), 1-50.

[11] D. Lewis and K. Mahler, Representation of integers by binary forms, Acta Arith. 6 (1961), 333-363.

[12] K. Mahler, An application of Jensen's formula to polynomials, Mathematika 7 (1960), 98-100.

[13] K. Mahler, An inequality for the discriminant of a polynomial, Michigan Math. J. 11 (1964), 257-262.

[14] K. Mahler, Zur Approximation algebraischer Zahlen III, Acta Math. 62 (1934), 91-166.

[15] M. Mignotte, Sur un théorème de M. Langevin, Acta Arith. 54 (1989), 81-86.

[16] J. Mueller, Counting solutions of $\left|a x^{r}-b y^{r}\right| \leq h$, Quart. J. Math. Oxford (2) 38 (1987), 503-513.

[17] J. Mueller and W. M. Schmidt, Trinomial Thue equations and inequalities, J. Reine Angew. Math. 379 (1987), 76-99.

[18] J. Mueller and W. M. Schmidt, Thue's equation and a conjecture of Siegel, Acta Math. 160 (1988), 207-247.

[19] W. M. Schmidt, Thue equations with few coefficients, Trans. Amer. Math. Soc. 303 (1987), 241-255.

[20] J. L. Thunder, The number of solutions to cubic Thue inequalities, Acta Arith. 66 (1994), no. 3, 237-243.

[21] J. L. Thunder, On Thue inequalities and a conjecture of Schmidt, J. Number Theory 52 (1995), 319-328.

[22] J. L. Thunder, Decomposable form inequalities, Ann. of Math. (2) 153 (2001), no. $3,767-804$. 
School of Mathematics, Tata Institute of Fundamental Research, Homi Bhabha Road, Mumbai - 400 005, INDIA.

E-mail address: saradha@math.tifr.res.in, divyum@math.tifr.res.in 